\title{
Biomolecules from Snail mucus (Helix aspersa) conjugate Gold nanoparticles, exhibiting potential wound healing and anti-inflammatory activity
}

\author{
Jennifer Gubitosa ${ }^{1}$, Vito Rizzi ${ }^{2}$, Paola Fini ${ }^{1}$, Anna Laurenzana ${ }^{3}$, Gabriella Fibbi ${ }^{3}$, Clara \\ Veiga-Villauriz ${ }^{4}$, Fiorenza Fanelli ${ }^{5}$, Francesco Fracassi ${ }^{2}$, Alberto Onzo $^{6}$, Giuliana Bianco ${ }^{6}$, \\ Carmine Gaeta ${ }^{7}$, Antonio Guerrieri ${ }^{6}$, and Pinalysa Cosma ${ }^{2}$ \\ ${ }^{1}$ IPCF-CNR UOS di Bari \\ ${ }^{2}$ Università degli Studi di Bari Aldo Moro \\ ${ }^{3}$ Università degli Studi di Firenze \\ ${ }^{4}$ The University of Manchester Faculty of Medical and Human Sciences \\ ${ }^{5}$ Istituto di Nanotecnologia Sede di Bari \\ ${ }^{6}$ Università degli Studi della Basilicata Dipartimento di Scienze \\ ${ }^{7}$ Università degli Studi di Salerno Facoltà di Scienze Matematiche Fisiche e Naturali
}

June 9,2020

\begin{abstract}
In this work, for the first time, snail slime from garden snails "Helix Aspersa Müller", has been used to induce the formation of eco-friendly gold nanoparticles (AuNPs-SS), suitable for biomedical applications. An AuNPs-SS comprehensive investigation was performed observing AuNPs with an average particle size of $14 \pm 6 \mathrm{~nm}$, stabilized by a slime snail-based organic layer. Indeed, as recognised in high-resolution MALDI-MS analyses, and corroborated by FESEM, UV-Vis, ATR-FTIR and XPS results, it was possible to assess the main presence of peptides and amino acids as main components of the slime, that, combined with the AuNPs confers them interesting properties. More specifically, we tested, in vitro, the AuNPs-SS safety in human keratinocytes and their potential effect on wound healing as well as their anti-inflammatory properties in Murine Macrophages. Moreover, the AuNPs-SS treatment resulted in a significant increase of the urokinase-type plasminogen activator receptor (uPAR), essential for keratinocyte adhesion, spreading and migration, together with the reduction of LPS-induced IL1- $\beta$ and IL- 6 cytokine levels, and completely abrogated the synthesis of the inducible nitric oxide synthase (iNOS).
\end{abstract}

\section{INTRODUCTION}

The human body has different ways for protecting itself against potential injuries and infections, by means of the skin characteristics acting as an effective barrier. The physiological process of tissue repair at an injured site is the wound healing, which represents a complex integrated system of biological and molecular processes, such as cell migration, proliferation, extracellular matrix deposition and remodelling.[1] Under normal conditions, the healing process cascade is initiated by an inflammatory phase yielding to granulation tissue accumulation and scar remodelling after epithelial wound closure has been accomplished.[2] A normal wound healing process is constituted by various phases: coagulation (hemostasis), inflammation, cellular migration and proliferation, protein synthesis and wound contraction (fibroplasia), and scar formation (remodelling). Among these phases, three are the most important for a successful wound healing: fibroplasia, angiogenesis and re-epithelialisation. A delay in the development of these mechanisms leads to failure or prolong wound healing process with a crucial part attributable to the wound neovascularisation. [3,4] However, any change 
or pathological problem (for example ischemia, diabetes mellitus and so on) may result in the formation of chronic wounds difficult to heal, due to the failure of the normal wound healing process. [3,5] Another very important objective of the wound treatment is the inhibition of all the pathogenic organisms able to cause serious infection, by accelerating the healing process with reducing cicatrices and pain to the patients of all ages.[5] In fact, any delay during the tissue repair process could be defined as chronic injury and can have a highly detrimental impact on human health being more subjected to pathogens. Rapid closure of dermatological wounds is of vital importance in preventing infection and reducing post-treatment side-effects.[6] So, shortening the chronic wound healing process is a prime challenge in modern dermatology.[7]

Several different topical and systemic agents, together with the antimicrobial ones have been developed for improving the cure of surgical and traumatic wounds. However, these functional topical biomaterials might delay re-epithelialisation and might result a risk for oversensitivity and resistance.[4] Hence, some alternative wound treatment methods have been searched for and, in this regard, the use of nanotechnology could be useful as a prominent scientific discipline in the technological revolution of this millennium. [8] Nanobiotechnology-based therapies in the field of wound healing are considered to be a promising area with new benefits based on the utilization of bionanomaterials in promoting wound healing, including antibacterial, antiinflammatory, regulating extracellular matrix production, promoting stem cell proliferation and differentiation, and enhancing growth factors.[9] Researches using gold nanoparticles (AuNPs) of different sizes and morphologies have been proposed in biomedical applications for skin injury therapy with great potential.[6] AuNPs have received great attention due to their low toxicity to animal and microorganism cells compared to other metal nanoparticles,[10] promoting their use as drug delivery systems, markers, and photothermal agents, as well as radiotherapy enhancers as well.[6] Recent studies have established new prospects for developing properly functionalized AuNPs for wound healing, acting as triggers to activate proliferation and migration of wound cells. $[7,11,12]$ The AuNPs use should exhibit benefits including enhanced mechanical stability and resistance against enzymatic degradation when incorporated into tissue scaffolds, in presence of antibodies, growth factors, and peptides, rendering them promising candidates for skin tissue engineering applications. [8]

Chemical methods, in which reduction or co-precipitation processes take place for the generation of nanoparticles, have been investigated since ancient times. However, the main drawbacks of these synthetic routes are the use of harsh chemical conditions of work, organic solvents and the production of toxic by-products during the synthesis and/or functionalization of the nanoparticles. For this purpose, many studies are focused on finding alternative therapeutic approaches by adopting biocompatible, safer and greener methods employing plant extracts for the fast and economical synthesis of biogenic nanogold.[13-15] Green synthesized AuNPs can also exhibit anticancer and antimicrobial activity,[16] antioxidant moisture retention, skin lightening properties,[17] and sunscreen ability, enlarging their applicability.[18,19] As reported by Gonnelli et al. ,[20] synthetic routes relying on green chemistry show other advantages for large scale production adopting mild conditions of work.[20] For green synthesized AuNPs, additional assembling steps are not necessary since the AuNPs properties are ascribed to main components present on their surface and used to induce their formation. Thus, considering these factors, the snail secretion (SS) from Helix Aspersa, currently revolutionizing the world of cosmetics and human skin care, has been used in this work for synthesizing AuNPs (AuNPs-SS), conferring them interesting properties. Indeed, the SS protein content plays a key role in cell regeneration and growth, preventing the effect of the inflammatory disease.[21,22] The SS main compounds are used as reducing agents enabling the AuNPs synthesis via reduction of $\mathrm{Au}(\mathrm{III})$, from a $\mathrm{HAuCl}_{4}$ solution, to $\mathrm{Au}(0)$, while forming an organic layer around the AuNPs. AuNPs-SS are thus synthetized by using a single step reaction, avoiding the use of additional and toxic agents. The nanoconfined main components of the SS on the AuNPs surface could be an interesting way for improving the whole process, combining the properties of gold and SS. For example, Conte et al .[23] reported that the pharmacokinetics of snail components do not permit their optimal absorption, so the combined use of AuNPs, well-known antibacterial agents, and SS could be a functional ingredient in the treatment of acne and in biotechnology, or used as a preservative in cosmetic products formulation. Considering the overall literature published,[21,22,24-27] although interesting information can be found on the SS use, the presence of fragmentary and not detailed investigations suggests that 
this research field has not been extensively explored yet. In a time when cosmetic dermatology and beauty care are increasingly influential, $[28,29]$ we intend to discuss the biogenic formation of AuNPs-SS by characterizing them and by applying as accelerating wound healing and anti-inflammatory agents in biomedicine. More specifically, we tested the effect of AuNPs-SS on improving wound healing in human keratinocytes. Furthermore, considering that gold has been used historically in the treatment of painful inflammatory conditions, such as rheumatoid arthritis, and snail slime has been proved to clear up skin inflammation, we evaluated the potential anti-inflammatory properties of AuNPs-SS in Murine Macrophages.

\section{EXPERIMENTAL SECTION}

\subsection{Chemicals.}

$\mathrm{HAuCl}_{4}$, and $\mathrm{KCl}$ used for Zeta potential measurements were purchased from Sigma-Aldrich (Milan, Italy). The Snail Slime was received from the Società Agricola Dap, di Francesco Paolo Perrotta \& C. S.a.s - via Diego Rapolla, - 85029 Venosa (Potenza, Basilicata, Italy). NCTC2544 human keratynocytes and RAW 264.7 macrophages were obtained from American Type Culture Collection (ATCC, Rockville, MD) and maintained at $37^{\circ} \mathrm{C}$ in DMEM media (Eurclone) in a humidified $5 \% \mathrm{CO}_{2}$ incubator maintained at $37^{\circ} \mathrm{C}$ (Thermo Scientific, Waltham, MA). For RAW 264.7 cells, the complete media contained L-glutamine, $100 \mathrm{U}$ penicillin or streptomycin/ml, and 10\% inactivated fetal calf serum (FBS, Euroclone). In the LPS stimulated macrophage experiments, RAW 264.7 cells were pre-treated overnight with $1 \mu \mathrm{g}$ LPS $/ \mathrm{mL}$. The day after, the LPS was removed and the cells replaced with fresh media in presence of AuNPs-SS.

\subsection{Mechanical extraction of snail slime.}

The snail Helix Aspersa Muller is the terrestrial gastropod mollusk that in the adult phase has a shell of a $3.5 \mathrm{~cm}$ diameter for a weight of about $15 \mathrm{~g}$. Among mechanical methods that do not damage the animal, the use of a natural gas as ozone was selected to induce the production of snail slime. The mechanical extraction involved the use of an equipment that within a few hours allow to obtain the slime. More specifically, the snails were inserted into a machine in which ozone was nebulized for about an hour; the $\mathrm{O}_{3}$ generates a sort of excitement that induces the slime production avoiding the stress of the snail.

\subsection{AuNPs-SS synthesis.}

$\mathrm{HAuCl}_{4}$ was solubilized in deionized water to obtain a $1.0 \times 10^{-3}$ stock solution. $1.5 \mathrm{~mL}$ of this solution were mixed with $2 \mathrm{~mL}$ of water and $500 \mu \mathrm{L}$ of the received SS. The mixture was moderately stirred at room temperature. The UV-Vis spectra of this solution were carried out soon after the preparation and at 4, 6, 24, 48 and $72 \mathrm{~h}$ after, to monitor the AuNPs-SS formation. The induced AuNPs-SS were purified at each acquisition time, and the excess SS and unreacted $\mathrm{HAuCl}_{4}$ were removed by washing in fresh water and centrifuging (7000 g, for 10 minutes). Before the spectra acquisition, the obtained solutions were properly diluted if necessary.

\subsection{UV-Visible measurements.}

The Visible absorption spectra of solutions were collected using a Varian CARY 5 UV-Vis-NIR spectrophotometer (Varian Inc., now Agilent Technologies Inc., Santa Clara, CA, USA) in the range 200-800 nm. Measurements were performed using a cuvette with a $1 \mathrm{~cm}$ path length.

\subsection{ATR-FTIR spectroscopic measurements.}

ATR-FTIR spectra were recorded in a $600-4000 \mathrm{~cm}^{-1}$ range, at a resolution of $4 \mathrm{~cm}^{-1}$, using a Fourier Transform Infrared spectrometer 670-IR equipped with an ATR device (Varian Inc., now Agilent Technologies Inc., Santa Clara, CA, USA). 32 scans were summed for each acquisition.

\subsection{Field Emission Scanning Electron Microscopy (FESEM).}

A Zeiss SUPRA 40 field emission scanning electron microscope was used for the morphological investigation of the AuNPs-SS. FESEM images were acquired using a high resolution in-lens secondary electron detector at the electron acceleration voltage (extra-high tension, EHT) of $20 \mathrm{kV}$, working distance of $4 \mathrm{~mm}$ and 
magnification in the range 100-1000k $\times$. The size distribution of AuNPs as well as the thickness of the organic layer covering the AuNPs was estimated by FESEM images. For FESEM and XPS investigation, AuNPs-SS were dispersed in water, deposited by drop-casting onto small pieces of a $\mathrm{Si}(100)$ wafer, and analyzed after solvent evaporation at room temperature.

\subsection{X-ray Photoelectron Spectroscopy (XPS).}

XPS analyses were performed using a PHI VersaProbe II spectrometer equipped with a monochromatic Al $\mathrm{K} \alpha \mathrm{X}$-ray source $(1486.6 \mathrm{eV})$ operated at a spot size of $100 \mu \mathrm{m}$ corresponding to a power of $24.5 \mathrm{~W}$. Survey $(0-1400 \mathrm{eV})$ and high resolution $(\mathrm{C} 1 \mathrm{~s}, \mathrm{O} 1 \mathrm{~s}, \mathrm{~N}$ 1s, Au 4f) spectra were recorded in FAT (fixed analyzer transmission) mode at pass energy of 117.4 and $46.95 \mathrm{eV}$, respectively. All spectra were acquired at a takeoff angle of $45^{\circ}$ with respect to the sample normal. Dual-beam charge neutralization was constantly applied during analysis. XPS analysis was repeated on three different spots of the sample. Special attention was devoted to verify that no change in the sample was induced by exposure to the $\mathrm{X}$-ray beam and to the dualbeam used for charge neutralization. Detailed spectra processing was performed by commercial MultiPak software (Version 9.5.0.8, 30-10-2013, Ulvac-PHI, Inc.). Charge correction of the spectra was performed by taking the hydrocarbon $(\mathrm{C}-\mathrm{C} / \mathrm{C}-\mathrm{H})$ component of the $\mathrm{C} 1 \mathrm{~s}$ spectrum as internal reference (binding energy, $\mathrm{BE}=285.0 \mathrm{eV}$ ). The high-resolution spectra were fitted with mixed Gaussian-Lorentzian peaks after a Shirley background subtraction. A maximum relative standard deviation of $10 \%$ was estimated on the area percentages of the curve-fitting components, while the determined standard deviation of their position was $\pm 0.2 \mathrm{eV}$.

\subsection{Zeta potential and size measurements.}

The average of the hydrodynamic diameters and the polydispersity index (PDI) of the AuNPs-SS were measured by dynamic light scattering and using a Zetasizer Nano ZS (Malvern Instruments Ltd., Worcestershire, UK). The surface charge of AuNPs-SS was measured as zeta potential by laser doppler velocimetry using the same instrument after dilution with $1 \mathrm{mM} \mathrm{KCl}$.

\subsection{Mass spectrometry analysis.}

High-resolution mass spectra were acquired on a Bruker (Bruker Daltonik GmbH, Bremen, Germany) solariX XR Fourier transform ion cyclotron resonance mass spectrometer (FT-ICR-MS) equipped with a 7T superconducting magnet and a MALDI source. Spectra were acquired with a Time Domain size of 16 megaword, an accumulation time of $0.1 \mathrm{~s}$ and a mass range of 100-2000 m/z. Moreover, the average number of scans was set to 50 . For the analysis, a laser power of $32 \%$ and a number of laser shots of 28 were assumed. 2,5-dihydroxybenzoic acid was used as a matrix, while a solution of this compound and pure snail slime (or AuNPs slime sample), in a 1:1 ratio, was prepared and analyzed. The mass measurement accuracy reached values of less than $0.1 \mathrm{ppm}$.

\subsection{Cell viability determination .}

The viability of NCTC2544 human keratinocyte and RAW 264.7 macrophages was determined by trypan blue staining. NCTC2544 cells $\left(2.0 \times 10^{5}\right)$ were seeded in 6 -well plates and allowed to attach overnight. On the next day AuNP colloidal solutions were added at the indicated concentrations. $48 \mathrm{~h}$ later $20 \mu \mathrm{l}$ of cells were aseptically transferred to a $1.5 \mathrm{~mL}$ clear Eppendorf tube and incubated for $3 \mathrm{~min}$ at room temperature with an equal volume of $0.4 \%$ (w/v) trypan blue solution prepared in $0.81 \% \mathrm{NaCl}$ and $0.06 \%$ (w/v) dibasic potassium phosphate. Viable and nonviable cells (trypan blue positive) were counted separately using a dual-chamber hemocytometer and a light microscope. RAW 264.7 cells $\left(3.0 \times 10^{5}\right)$ were seeded in 6 -well plates and allowed to attach overnight. On the next day cells were pre-treated overnight with $1 \mu \mathrm{g} \mathrm{LPS} / \mathrm{mL}$. The day after the LPS was removed and the cells replaced with fresh media in presence of AuNPs-SS. 24h later viable and non-viable cells were counted as described fo NCTC2544. The means of three independent cell counts were pooled for analysis.

\subsection{Scratch assay.}


The migratory potential of the keratinocyte cell line NCTC2544 was assessed with a wound healing scratch assay.[30] NCTC cells were grown to confluence in $60 \mathrm{~mm}$ culture plates at a concentration of $2 \times 10^{5}$ cells in complete medium. A straight scratch was made in the confluent monolayer with a p200 pipet tip. Detached cells were removed, and the edge of the scratch was flattened by washing the cells twice with PBS. Cells were replaced with complete media and treated with increasing concentration of AuNPs-SS to monitor cell motility. Scratched areas for each sample were marked and photographed immediately and after 24 and 48 hours with an EVO digital camera (Coolpix 5400, Nikon Corporation, Japan). Using the ImageJ image processing program, the size of the denuded area was determined at each time point from the digital images. The migrated area was calculated by subtracting the wound area at time point $t=24$ hours and $\mathrm{t}=48$ hours from $t=0 \mathrm{~h}$.

\subsection{RNA extraction, semiquantitative and quantitative PCR .}

Total RNA was prepared using Tri Reagent (Sigma-Aldrich, Saint Louis, Missouri, USA), agarose gel checked for integrity, and reverse transcribed with cDNA synthesis kit (BioRad, Milano, Italy) according to manufacturer's instructions. Selected genes were evaluated by qualitative PCR using Blue Platinum PCR Super Mix (Life Technologies, Monza, Italy). Primer sequences (IDT, TemaRicerca, Bologna, Italy) were as follows:

IL1- $\beta$ Forward 5'-CCTGCAGCTGGAGAGTGTGGA-3'; Reverse 5'-AGGAGGAACGGA-GACTACCC-3'

iNOS Forward 5' -CCC TTC CGA AGT TTC TGG CAG CAG C-3'; Reverse 5'-GGC TGT CAG AGC CTC GTG GCT TTG-3'

IL6 Forward 5'- CGG AGA GGA GAC TTC ACA CAG GA-3' Reverse 5'- GGA GAG CAT TGG AAA TTG GGG-3'

$\beta$-actin Forward 5' GGC ACC ACA CCT TCT ACA ATG-3' Reverse 5'- GGG GTG TTG AAG GTC TCA AAC - 3'

\subsection{Western blot analysis.}

Harvested cells were resuspended in 20 mM RIPA buffer (pH 7.4) (Merk Millipore, Vimodrone, MI, Italy) containing a cocktail of proteinase inhibitors (Calbiochem, Merck, Darmstadt, Germany) and treated by sonication 19 (Microson XL-2000, Minisonix, Farmingdale, NY, USA). Aliquots of supernatants containing equal amounts of protein $(30 \mu \mathrm{g})$ in Laemmli buffer were separated on Bolt@ B Bis-Tris Plus gels 4-12\% precast polyacrylamide gels (Life Technologies, Monza, Italy) and then transferred from the gel to a PVDF nitrocellulose membrane using iBlot 2 system (Life Technologies, Monza, Italy). Blots were stained with Ponceau red to ensure equal loading and complete transfer of proteins. Then, they were blocked for 1 hour, at room temperature, with $5 \%$ milk in PBS $0.1 \%$ tween solution. Subsequently, the membranes were probed at $4^{\circ} \mathrm{C}$ overnight with the following primary antibodies: rabbit anti-uPAR (1:500 FL 290, Santa Cruz Biotechnology, Cat\# sc-10815); rabbit anti-iNOS (1:1000, Cell signaling, Cat\#2982) rabbit GAPDH antibody (1:1000, Cell signaling Technology, Cat\# 2118) was used to assess equal amount of protein loaded in each lane. Anti-Rabbit IgG (whole molecule)-Peroxidase antibody (Sigma, Cat\#A0545) was used as the secondary antibody; the ECL procedure was employed for development.

\section{RESULTS AND DISCUSSION}

After mixing, under continuous stirring, appropriate amounts of SS and $\mathrm{HAuCl}_{4}$, the formation of AuNPs-SS was observed. More specifically, by diluting the received SS with a dilution factor of 1:8, fixing the $\mathrm{HAuCl}_{4}$ concentration at $4.0 \times 10^{-4} \mathrm{M}$, the colour of the reaction mixture changed from light yellow (Figure 1A , picture 1) to light purple (Figure 1A , picture 2). In particular, extending the contact time to $48 \mathrm{~h}$, the colour of these solutions strikingly changed to dark purple indicating the AuNPs-SS formation. As suggested by Yin et al. ,[31] the optical properties of nanostructured gold depend strongly on the size, shape, interactions between the nanoparticles and the species adsorbed on their surface. The colour of the colloidal gold is due to the surface plasmon resonance (SPR) absorption, a characteristic signal in the visible absorption spectrum of AuNPs, in general showed in the range from 500 to $600 \mathrm{~nm}$ in the UV-Vis absorption spectrum. In this 
case, the mixture of $\mathrm{HAuCl}_{4}$ and SS presents the AuNPs characteristic SPR band, that appeared slightly broadened and centered at $550 \mathrm{~nm}$ (Figure 1B ). In particular, inFigure 1B, the AuNPs SPR band time evolution is reported. While in Figures $\mathbf{1 C}$ and $\mathbf{1 D}$, the wavelength and absorption values at the maximum of the SPR are showed as function of time compared with the Full Width at Half Maximum (FWHM). A shift towards higher wavelength number values, along with an increase of the $\mathrm{A}_{\max }$ were observed by extending the contact time. At the same time, the corresponding FWHM became larger than values observed at the beginning of the reaction (Figures 1C, D ). Always according Yin et al. ,[31] the variances in the SPR bands can be attributed to a different distribution of the nanoparticles in the solution and to their aggregation. Moreover, the SPR absorption intensity of AuNPs depends on their size and shape as well as their surrounding medium.[31]

Therefore, in agreement with the SPR band position and shape, the spectral observations indicated polydispersed AuNPs that tend to slightly increase their size with elapsing the contact time. Probably, the observed increment in the $\mathrm{A}_{\max }$, passing from 4 to 48h, could be attributed to an increase in the AuNPs-SS concentration due to the quite complete reaction of $\mathrm{HAuCl}_{4}$ with SS. Further, it is worth mentioning that, incrementing the contact time until $72 \mathrm{~h}$, the particles tended to aggregate to each other and the SPR signal decreased, becoming barely evident in the UV-Vis spectrum (data not shown ).

As a whole, the results indicated the reduction of $\mathrm{Au}(\mathrm{III})$ to $\mathrm{Au}(0)$, forming nanoparticles thanks to the synergistic action, as reducing agents, of main components of snail mucus. Indeed, the chemical reduction of $\mathrm{HAuCl}_{4}$ aqueous solution is one of the most widely used methods for the synthesis of colloidal gold. [32] Not surprisingly, by looking at the AuNPs-SS spectrum shape line, in particular below $400 \mathrm{~nm}$, the contribution of chemical species, like proteins/peptides and amino acids, mainly present in SS, [33] absorbing in this region, was observed. The finding was confirmed by comparing the UV-Vis spectrum of SS solutions with the AuNPs spectrum (Figure S1). The UV-Vis spectra of SS collected at different dilutions (to better evidence the contribution of the absorbance values in the reported wavelength region) clearly displayed the contribution of the main SS components, indicated with arrows, on the AuNPs surface.

A Zeta potential value of approximately $-35 \pm 2 \mathrm{mV}$ was measured for AuNPs-SS, indicating their good stability in water. Further,Figure S2 shows the comparison between the UV-Vis spectra of AuNPs-SS collected soon after the synthesis (selecting $24 \mathrm{~h}$ as contact time) and after 2 months. It is clearly evident as AuNPs-SS are characterized by a long-time stability, as indicated by the presence of the AuNPs SRP signal.

\subsection{FESEM Analysis}

FESEM investigation revealed the formation of irregularly shaped AuNPs (Figure 2A, B ), with minimum and maximum size of about 4 and $50 \mathrm{~nm}$, respectively, and average size of $14 \pm 6 \mathrm{~nm}$ (particles size distribution in Figure 2C ). FESEM images confirmed the hybrid organic-inorganic nature of the AuNPs-SS and, in particular, evidenced the presence of a thin organic layer covering the Au nanoparticles (thickness ranging between 4 and 8 nm, Figure 2B ).

So, starting from these considerations and considering an AuNPs-SS average size of $14 \pm 6 \mathrm{~nm}$, a molar absorption coefficient of $3.7 \times 10^{8} \mathrm{M}^{-1} \mathrm{~cm}^{-1}$ was calculated. As a result, each synthesis produced, adopting $24 \mathrm{~h}$ as contact time, AuNPs-SS stock solutions with a mean concentration of $5 \times 10^{-9} \mathrm{M}$. It means $1.64 \times 10^{12} \mathrm{AuNPs} / \mathrm{mL}$.

\subsection{XPS analysis}

XPS results showed that the surface atomic concentrations of carbon, oxygen, nitrogen and gold are about 64, 18, 11 and 7\%, respectively.Figure 3A reports the high-resolution Au 4f XPS spectrum consisting of a doublet (i.e., $\mathrm{Au} 4 \mathrm{f}_{7 / 2}$ and $\mathrm{Au} 4 \mathrm{f}_{5 / 2}$ at 83.2 and $86.9 \mathrm{eV}$, respectively) due to spin-orbit coupling, the position being in agreement with literature on AuNPs.[18,34-37] The curve fitting of the Au $4 \mathrm{f}$ signal indicates three $\mathrm{Au} 4 \mathrm{f}_{7 / 2}$ components, i.e., the main component at $83.2 \mathrm{eV}(68 \%)$ attributed to metallic gold $(\mathrm{Au}(0))$, and the minor peaks at about $84.2 \mathrm{eV}(23 \%)$ and $85.4 \mathrm{eV}(9 \%)$ ascribed to $\mathrm{Au}(\mathrm{I})$ and $\mathrm{Au}(\mathrm{III})$, respectively (the total area of the $\mathrm{Au} 4 \mathrm{f}_{7 / 2}$ is taken as 100\%). [37-40] The high-resolution C1s XPS spectrum inFigure 3B is curve-fitted with four peaks and, specifically, the hydrocarbon component at $285 \mathrm{eV}$ (51\%), the component 
at $286.4 \mathrm{eV}(31 \%)$ ascribed to both $\mathrm{C}-\mathrm{N}$ and $\mathrm{C}-\mathrm{O}$ groups, the peak at $288.0 \mathrm{eV}(14.5 \%)$ assigned to both $\mathrm{C}=\mathrm{O}$ and O-C-O moieties and the weak peak at $289.2 \mathrm{eV}(3.5 \%)$ due to carboxylic functionalities. The O1s spectrum inFigure 3C displays two components, i.e., the peak at $531.7 \mathrm{eV}(57 \%)$ that can be ascribed to $\mathrm{O}=\mathrm{C}$ moieties and the component at $533.1 \mathrm{eV}(43 \%)$ associated with O-C groups. The N1s signal (Figure 3D ) shows one main peak at about $400.1 \mathrm{eV}$, consistent with the presence of amine and amide groups. [41-43] To unveil the nature of these compounds on the AuNPs surface, ATR-FTIR and MALDI-MS analyses were performed.

\subsection{ATR-FTIR spectroscopic measurements}

FTIR measurements were carried out to recognize the possible functional groups responsible for efficient stabilization of gold nanoparticles prepared by using SS. For this purpose, a comparison between the ATRFTIR spectra of SS before and after the formation of AuNPs, and the FTIR spectrum of AuNPs-SS was performed (Figure 4 ).

Starting from as received SS, bands around $1700 \mathrm{~cm}^{-1}$ and $1580 \mathrm{~cm}^{-1}$ were detected, and in accordance with literature, $[33,44,45]$ these peaks indicated the main presence of amide bonds of peptides and proteins as glycosoaminoglycans and proteoglycans. Further, the bands at about $1388 \mathrm{~cm}^{-1}$ and $1700 \mathrm{~cm}^{-1}$ could suggest the presence of carboxylate moieties associated with acid derivates of sugars and amino acids chains. Indeed, the peaks at $1192 \mathrm{~cm}^{-1}$ and at lower wavenumber values (below $900 \mathrm{~cm}^{-1}$ ) indicated the O-glyosidic linkages.[33] In this region, the contribute of sulphated, acylated and ester based compounds should be also taken into account. The shoulder at $1360 \mathrm{~cm}^{-1}$ with a partial contribute at $1380 \mathrm{~cm}^{-1}$ indicated the presence of carbon chains of proteins and carbohydrates. [33,44,45] Accordingly, there was evidence at $2850 \mathrm{~cm}^{-1}$ and $2930 \mathrm{~cm}^{-1}$ of $\mathrm{CH}_{2}$ and $\mathrm{CH}_{3}$ arisen from protein skeleton and lipidic component of the slime.[30] The observed bands between 2900 and $3500 \mathrm{~cm}^{-1}$ can be ascribed to the presence of a large amount of $\mathrm{OH}$ and $\mathrm{NH}$ moieties. The contributes of polyphenols, mainly in the range 1500-3500 $\mathrm{cm}^{-1}$, cannot be excluded.[18] Overtones of the benzene rings of phenylaniline and tyrosine were detected above $1800 \mathrm{~cm}^{-1}$ and $2600 \mathrm{~cm}^{-1}$.[33] Interestingly, after the contact with $\mathrm{HAuCl}_{4}$ solution, and thus after the AuNPs formation, the SS spectrum appeared almost unchanged as a whole in comparison with the one observed at the beginning of the reaction. In particular, the signal at $1580 \mathrm{~cm}^{-1}$ disappeared, but others main vibrations at $1700 \mathrm{~cm}^{-1}$ and $1192 \mathrm{~cm}^{-1}$ were retained. Then, if, on one hand, the disappearance of the of amide bond signals from peptides and proteins features was indicative of their role in the formation of AuNPs-SS, on the other hand, the counterpart of the spectrum ascribed to sugar derivates did not show important changes. The wavenumber region 2800-3500 $\mathrm{cm}^{-1}$ appeared also affected, suggesting the contribute of biomolecules having $\mathrm{OH}$ and $\mathrm{NH}$ functionalities. At the same time, the bands intensity at $2850 \mathrm{~cm}^{-1}$ and $2930 \mathrm{~cm}^{-1}$ was reduced further suggesting the role of proteins in nanoparticles formation. These results were confirmed when the FTIR-ATR of the AuNPs-SS was collected. Indeed, the main signals at 1645 and $1545 \mathrm{~cm}^{-1}$, indicated the presence of a protein component based on the Amide I (C=O stretching) $\left(1645 \mathrm{~cm}^{-1}\right)$ and II $\left(1545 \mathrm{~cm}^{-1}\right)(\mathrm{N}-\mathrm{H}$ bending and $\mathrm{C}-\mathrm{N}$ stretching $)$ bands. [44] Interestingly, if these bands were compared with signals of SS and ascribed to the same vibration modes, the observed shift towards lower wavenumber values could be attributed to their coordination on the AuNPs surface. The characteristic absorption in the region $1200-1300 \mathrm{~cm}^{-1}$, which represented the amide III, due to the $\mathrm{C}-\mathrm{N}$ stretching and $\mathrm{N}-\mathrm{H}$ deformation bands, was also detected. Accordingly, signals between 3300 and $3750 \mathrm{~cm}^{-1}$ arise also from the AuNPs-SS surface due to $\mathrm{N}-\mathrm{H}$ amine group stretching vibrations superimposed on the side of $\mathrm{O}-\mathrm{H}$ hydroxyl group stretching bands.[46] Once again, comparing the position of these bands with those observed in the as received SS, a clear shift towards higher wavenumbers was observed confirming that the molecules in the SS could coordinate to AuNPs through formation of Au-N or $\mathrm{Au}-\mathrm{O}$ covalent bonds. In the spectral region from 750 to $1400 \mathrm{~cm}^{-1}$ only very weak signals can be observed. The lacking of clear peaks at 1192, $1388 \mathrm{~cm}^{-1}$ and $1700 \mathrm{~cm}^{-1}$ could suggest the absence O-glycosidic linkages and carboxylate moieties associated with acid derivates of sugars and amino acids chains. The signal at 2850 $\mathrm{cm}^{-1}$ and $2930 \mathrm{~cm}^{-1}$ observed in the ATR-FTIR of AuNPs-SS could confirm the presence of protein skeleton. So, from the ATR-FTIR analyses it has been possible to assess the main presence of peptides and aminoacids on the surface of AuNPs-SS, acting as reductant agents during the formation of AuNPs. 


\subsection{MALDI (+) FT-ICR MS Analysis.}

The presence of amino acids and peptides was supported by MALDI (+) FT-ICR MS experiments (Figure 5 ). The MALDI (+) FT-ICR MS spectrum for as received SS sample (Figure 5A ) shows a huge number of MS signals, thus revealing the complexity of the sample in terms of its metabolic profile. Thanks to higher levels of accuracies reached by HRMS technique, [47] it was possible to unequivocally identify several protonated amino acids, i.e. threonine $\left(\left[\mathrm{C}_{4} \mathrm{H}_{10} \mathrm{NO}_{3}\right]^{+}, \mathrm{m} / \mathrm{z} 120.12747\right)$, tryptophan $\left(\left[\mathrm{C}_{11} \mathrm{H}_{13} \mathrm{~N}_{2} \mathrm{O}_{2}\right]^{+}, \mathrm{m} / \mathrm{z}\right.$ 205.23364), arginine $\left(\left[\mathrm{C}_{6} \mathrm{H}_{15} \mathrm{~N}_{4} \mathrm{O}_{2}\right]^{+}, \mathrm{m} / \mathrm{z} 175.20953\right)$, hystidine $\left(\left[\mathrm{C}_{6} \mathrm{H}_{10} \mathrm{~N}_{3} \mathrm{O}_{2}\right]^{+}, \mathrm{m} / \mathrm{z}\right.$ 156.16258), asparagine $\left(\left[\mathrm{C}_{4} \mathrm{H}_{9} \mathrm{~N}_{2} \mathrm{O}_{3}\right]^{+}, \mathrm{m} / \mathrm{z} 133.12607\right)$, cysteine $\left(\left[\mathrm{C}_{3} \mathrm{H}_{8} \mathrm{NO}_{2} \mathrm{~S}\right]^{+}, \mathrm{m} / \mathrm{z} 122.16670\right)$, glutamine $\left(\left[\mathrm{C}_{5} \mathrm{H}_{11} \mathrm{~N}_{2} \mathrm{O}_{3}\right]^{+}, \mathrm{m} / \mathrm{z}\right.$ 147.15341), proline $\left(\left[\mathrm{C}_{5} \mathrm{H}_{10} \mathrm{NO}_{2}\right]^{+}, \mathrm{m} / \mathrm{z}\right.$ 116.13903) and serine $\left(\left[\mathrm{C}_{3} \mathrm{H}_{7} \mathrm{NO}_{3}\right]^{+}, \mathrm{m} / \mathrm{z}\right.$ 106.10083), already found in snail slime sample.[48] 6198 peptides could be identified showing the presence of at least one unit of cysteine. The presence of peptides showing high contents of cysteine, proline, serine and glycine was accessed too, these ones very well-known in the literature for their high antimicrobial activity.[48] Instead, analysis of AuNPs-SS sample (Figure 5B ) shows a very different situation. In this case, MALDI (+) FT-ICR MS spectrum of the sample shows a lower number of MS signals in comparison to the pure sample (3158 and 209694 signals, respectively). No free amino acids were found and only 3 free peptides could be identified, thus suggesting a drastic shrinking of the range of free peptides present in the sample, indicative of their role in the formation of AuNPs.

\section{$3.5 \mathrm{pH}$ dependence of synthesized AuNPs.}

The AuNP stability, after their synthesis, was investigated under different $\mathrm{pH}$ values ranging from 2 to 12 . Once again, by observing the SPR of AuNPs, the wavelength and FWHM were monitored. Results, reported in Figure 6A , indicated that both the position and the FWHM were stable in the $\mathrm{pH}$ range from 4 to 10 and slight changes were detected at the lowest and the highest investigated $\mathrm{pH}$ value, i.e. $\mathrm{pH} 2$ and 12 . More specifically, at $\mathrm{pH} 2$ the SPR signal shift towards higher wavelength values indicating the contribution of larger AuNPs that under this condition tend to agglomerate.[31] Yinet al .[31] suggested that a large number of $\mathrm{H}^{+}$in the aqueous solution could be adsorbed onto the surface of the AuNPs, modifying their surface and their charges. Accordingly, at the same time, the FWHM increased. Then, AuNPs tended to agglomerate easily and consequently the final particle size was larger. Conversely, at $\mathrm{pH} 12$ the contribute of smaller AuNPs occurred in agreement with their stabilization, under this condition of work. The FWHM in this case reduced its value, indicating that the particles were more monodisperse. These findings were confirmed by performing Zeta potential measurements as function of $\mathrm{pH}$ values, as reported inFigure 6B . It is worth mentioning that the Zeta potential gives information about the AuNP suspension stability, since a higher electric surface charge of the AuNPs prevents aggregation. Indeed, the AuNPs were negatively charged in the range of $\mathrm{pH}$ from 4 to 12 and, in particular, the charge decreased at the increasing of the $\mathrm{pH}$ value. On the other hand, at $\mathrm{pH} 2$ a positive Zeta potential value, of approximately $+25 \mathrm{mV}$, was observed, affecting the AuNPs surface as previously described. The presence of functional groups arisen from the main components of SS, mainly the carboxylic moieties of proteins and aminoacids (and/or polyphenols) present on the AuNP surface, produced a negative electrical charge and the observed dependence on the $\mathrm{pH}$. At $\mathrm{pH}$ values below 4, the organic moieties were in the protonated form and the AuNPs slightly aggregated.

\subsection{AuNPs-SS enhance scratch wound closure of NCTC 2544 keratinocytes.}

To determine whether AuNPs-SS could affect cell motility, scratch assays were performed in a confluent monolayer of keratinocytes and evaluated at $0 \mathrm{~h}, 24 \mathrm{~h}$ and $48 \mathrm{~h}$ after treatment with increasing doses of AuNPs-SS. By 24h, even though the keratinocytes normally showed an active migratory phenotype, scratches treated with AuNPs-SS displayed a significant acceleration of wound closure as compared with the untreated (CTRL) wound (Figures 7A, B ). By 48h the scratch treated with the highest AuNPs dose was completely closed, thus showing a significant increase in closure rate from $24 \mathrm{~h}$ while no statistically significant change in cell viability was observed on AuNPs-SS treatment as shown inFigure 7C . Moreover, western blot analysis displayed that AuNPs-SS treatment induced a significant increase of the urokinase-type plasminogen activator receptor (uPAR), which is essential for keratinocyte adhesion, spreading and migration in vitro and in vivo (Figure 8C ). [49,50] 
3.7 AuNPs-SS reduce the inflammatory response of LPS-activated macrophages.

To further assess possible effects of the AuNPs-SS on biological inflammatory response, RAW 264.7 macrophages were pre-treated with LPS and the day after loaded with two doses of AuNPs-SS. Morphological changes and an inflammatory gene panel were evaluated at the end of the sequential treatments. As shown in Figure 8A, the mouse macrophage cells took up AuNP regardless of treatment with LPS. Cells without LPS treatment were more homogeneous in size, and AuNPs appeared to be dispersed throughout the cytoplasm. After LPS treatment, macrophages became activated as seen by variations in size. The higher the AuNP concentration, the more activated cells appeared, presenting extended pseudopods. It was found there was no statistically significant change in macrophage viability (Figure 8B ). Gene expression of cytokines IL1- $\beta$, IL-6 and inducible nitric oxide synthase (iNOS), showed that, in the absence of LPS treatment, there were moderate changes in cytokines expression after AuNPs-SS treatment. LPS induced a massive increase in cytokines and iNOS expression. Interestingly, the AuNPs-SS synergistically reduced these elevated cytokine levels and completely abrogated the synthesis of iNOS (Figure $\mathbf{8 C}$ ). The effect of AuNPs-SS on iNOS levels before and after LPS stimulation was confirmed also by western blot analysis (Figure 8D ). Altogether these results suggest that AuNPss-SS may have modulatory effects that could be beneficial in reducing inflammatory or pathogenic responses.

\section{CONCLUSIONS}

For the first time, during this work, the Snail Slime was used to induce the formation of AuNPs, proposing in literature a novel and green approach for their synthesis. A comprehensive investigation was performed in our laboratory by adopting several complementary techniques such as UV-Vis and ATR-FTIR spectroscopy, DLS, XPS, and high-resolution MALDI-MS analyses. AuNPs $14 \pm 6$ wide were observed through the FESEM investigation that evidenced the formation of the organic layer Snail Slime-based surrounding AuNPs. The latter was confirmed by ATR-FTIR, XPS and MALDI-MS analyses. Indeed, the main presence of peptides and aminoacids was observed at the surface of the AuNPs giving them a high Zeta potential value in water $(-35 \pm 2 \mathrm{mV})$, indicative of their great stability. Indeed, the temporal and $\mathrm{pH}$ stability of the proposed colloidal gold solution was investigated, which resulted in a suspension stable for a long time and appeared only slightly affected under hard acidic $\mathrm{pH}$ value ( $\mathrm{pH} 2$ ), far from biological conditions, in which aggregation of AuNPs was observed. With the prospect of using the AuNPs for biomedical devices, we studied the effect of AuNPs on wound healing in human keratinocytes and evaluated their potential anti-inflammatory properties in Murine Macrophages. Our findings demonstrated that the accelerated wound closure promoted by AuNPs-SS was associated with an increased expression of the receptor for urokinase (uPAR) which, besides that canonical conversion of plasminogen to plasmin and extracellular matrix (ECM) degradation, directly controls cell adhesion, differentiation, proliferation and migration through non-proteolytic mechanisms.[49,50]

It has been established that in the classic process following infection or injury, macrophages play a key role as regulators of inflammation. However, if the inflammatory response of macrophages is not properly controlled, it may lead to severe diseases such as atherosclerosis, [51] rheumatoid arthritis (RA), vascular injury and cancer.[52] Accordingly, to moderate inflammation, the macrophages have been targeted for specific ablation, inhibition of infiltration and reduction of the pro-inflammatory cytokines release. In this work, we demonstrated for the first time the AuNPs-SS were able to modulate the inflammatory response induced by LPS in murine macrophages by significantly reducing the IL1- $\beta$, IL- 6 cytokines levels. It is known that macrophages isolated from chronic wounds in diabetic patients exhibit a proinflammatory phenotype with the release of IL-1 $\beta$, MMP-9, TNF- $\alpha$, and IL-6. In a recent study Mirza and coworkers[53] showed that IL-1 $\beta$ sustains the proinflammatory macrophage phenotype observed in poorly healing wounds of humans and mice. The authors of the study demonstrated that targeting the IL- $1 \beta$ pathway upregulated expression of pro-healing factors in wounds of diabetic mice and improved healing of these wounds. Indeed Sumbayev et al. reported that citrate-stabilized gold nanoparticles were able to down-regulate the inflammatory process by selectively targeting the IL-1 $\beta$ dependent pathway[54].

We also observed a massive drop of the inducible nitric oxide synthase (iNOS) levels, which is essential for the macrophage activation and thus for cytokines release. Regulation of iNOS gene expression is, through 
transcriptional regulation, particularly influenced by NF- $x \mathrm{~B}$ activation. Indeed, in mice, iNOS gene promoter contains two NF- $x$ B binding sites, both of which need to be bound in order to get full induction of iNOS by LPS stimulation

Hence, a novel horizon in the field of biotechnology is opened with this work, proposing an innovative nanoplatform that should have other properties, under investigation in our research groups. The applications in cosmetic formulations of AuNPs as an additional ingredient would give the possibility to avoid, for example, the use of preservatives, due to the potential antimicrobial activity of gold that, at least, should also preserve the snail slime components.

In conclusion, our findings demonstrated that AuNPs-SS promote effective wound healing and may pave the way to accelerate the regeneration of difficult-to-heal wounds. A more in-depth understanding of the effects of NPs on macrophage activation may lead to a more potent and efficient control of inflammatory-related diseases.

Acknowledgements

We gratefully acknowledge Mr. Sergio Nuzzo for the skillful and excellent technical assistance, and Francesco Paolo Perrotta and Francesca D'Andretta, Società Agricola Dap di Francesco Paolo Perrotta \& C. S.a.s - via Diego Rapolla, - 85029 Venosa (Potenza, Basilicata, Italy), for the snail slime production and Patrizia Iannece for her technical support, provided for Mass Spectrometry analysis. This research was supported by the Italian Ministry for Education, University and Research (MIUR) under grant PONa3_00369 - "Laboratorio SISTEMA".

\section{References}

[1] Thangavel, P., Kannan, R., Ramachandran, B., Moorthy, G., Suguna, L., Muthuvijayan, V. (2018). Development of reduced graphene oxide (rGO)-isabgol nanocomposite dressings for enhanced vascularization and accelerated wound healing in normal and diabetic rats, J. Colloid Interf. Sci. 517, 251-264. doi:10.1016/j.jcis.2018.01.110.

[2] Singer, A. J., Clark, R. A. F. (1999). Cutaneous wound healing.New Eng J Med, 341, 738-746. doi:

\subsection{6/NEJM199909023411006.}

[3] Shanmugapriya, K., Kang, H. W. (2019). Engineering pharmaceutical nanocarriers for photodynamic therapy on wound healing: Review. Mater. Sci. Eng. C, 105, 110110. doi:10.1016/j.msec.2019.110110.

[4] Rajendran, R., Volova, T., Oluwafemi, O. S., Thomas, R. S. S., Kalarikkal, N. (2020). Nano formulated proanthocyanidins as an effective wound healing component. Mater. Sci. Eng. C, 106, 110056.doi: 10.1016/j.msec.2019.110056.

[5] Menke, N. B., Ward, K. R., Witten, T. M., Bonchev, D. G., Diegelmann, R. F. (2007). Impaired wound healing. Clin Dermatol., 25(1), 19. doi: 10.1016/j.clindermatol.2006.12.005.

[6] Kim, J. E., Lee, J., Jang, M., Kwak, M. H., Go, J., Kho, E. K., Song, S. H., Sung, J. E., Lee, J., Hwang, D. Y. (2015). Accelerated healing of cutaneous wounds using phytochemically stabilized gold nanoparticle deposited hydrocolloid membranes. Biomater. Sci., 3 , 509-519. doi:10.1039/C4BM00390J.

[7] Pan, A., Zhong, M., Wu, H., Peng, Y., Xia, H., Tang, Q., Huang, Q., Wei, L., Xiao, L., Peng, (2018). Topical Application of Keratinocyte Growth Factor Conjugated Gold Nanoparticles Accelerate Wound Healing.Nanomed-Nanotechnol, 14(5) , 1619-1628, doi:10.1016/j.nano.2018.04.007;

[8] Ovais, M., Ahmad, I., Khalil, A. T., Mukherjee, S., Javed, R., Ayaz, M., Raza, A., Shinwari, Z. K. (2018). Wound healing applications of biogenic colloidal silver and gold nanoparticles: recent trends and future prospects. Appl. Microbiol. Biot., 102, 4305-4318.doi: 10.1007/s00253-018-8939-z

[9] Du, J., Wong, K.K.Y. (2019). Cap. 9 - Nanomaterials for Wound Healing: Scope and Advances in Theranostic Bionanomaterials . Elsevier, pp. 211-230. doi: 10.1016/B978-0-12-815341-3.00009-2. 
[10] Barai, A. C., Paul, K., Dey, A., Manna, S., Roy, S., Bag, B. G., Mukhopadhyay, C. (2018). Green synthesis of Nerium oleander-conjugated gold nanoparticles and study of its in vitro anticancer activity on MCF-7 cell lines and catalytic activity.Nano Convergence, 5, 10. doi: 10.1186/s40580-018-0142-5.

[11] Rajendran, N. K., Kumar, S. S. D., Houreld, N. N., Abrahamse, H. (2018). A review on nanoparticle based treatment for wound healing.J Drug Deliv Sci Tec, 44, 421-430. doi:10.1016/j.jddst.2018.01.009;

[12] Leu, J.G., Chen, S.A., Chen, H.M., Wu, W.M., Hung, C. F., Yao, Y.D., Tu, C. S., Liang, Y. J. (2012). The effects of gold nanoparticles in wound healing with antioxidant epigallocatechin gallate and alpha-lipoic acid. Nanomed-Nanotechnol, 8(5), 767-775.doi: 10.1016/j.nano.2011.08.013.

[13] Molnár, Z., Bódai, V., Szakacs, G., Erdélyi, B., Fogarassy, Z., Sáfrán, G., Varga, T., Kónya, Z., TóthSzeles, E., Szücs, R., Lagz, I. (2018). Green synthesis of gold nanoparticles by thermophilic filamentous fungi. Sci. Rep.-UK, 8, 3943. doi:10.1038/s41598-018-22112-3.

[14] Ismail, E.H., Saqer, A.M.A., Assirey, E., Naqvi, A., Okasha, R.M. (2018). Successful Green Synthesis of Gold Nanoparticles using a Corchorus olitorius Extract and Their Antiproliferative Effect in Cancer Cells. Int. J. Mol. Sci., 19, 2612. doi:10.3390/ijms19092612.

[15] Ghodake, G., Eom, C.-Y., Kim, S.W., Jin, E. (2010). Biogenic Nano-Synthesis; towards the Efficient Production of the Biocompatible Gold Nanoparticles. Bull. Korean Chem. Soc., 31, 2771-2775. doi: 10.5012/bkcs.2010.31.10.2771.

[16] Vijayan, R., Joseph, S., Mathew, B. (2018). Indigofera tinctoria leaf extract mediated green synthesis of silver and gold nanoparticles and assessment of their anticancer, antimicrobial, antioxidant and catalytic properties. Artif Cells Nanomed Biotechnol, 46(4), 861-871. doi: 10.1080/21691401.2017.1345930.

[17] Jiménez-Pérez, Z. E., Singh, P., Kim, Y.J., Mathiyalagan, R., Kim, D. H., Lee, M. H., Yang, D. C. (2018). Applications of Panax ginseng leaves-mediated gold nanoparticles in cosmetics relation to antioxidant, moisture retention, and whitening effect on B16BL6 cells.J Ginseng Res, 42(3), 327-333. doi:10.1016/j.jgr.2017.04.003.

[18] Gubitosa, J., Rizzi, V., Lopedota, A., Fini, P., Laurenzana, A., Fibbi, G., Fanelli, F., Petrella, A., Laquintana, V., Denora, N., Comparelli, R., Cosma, P. (2018). One pot environmental friendly synthesis of gold nanoparticles using Punica Granatum Juice: A novel antioxidant agent for future dermatological and cosmetic applications.J. Coll. Interf. Sci., 521, 50-61. doi:10.1016/j.jcis.2018.02.069.

[19] Borase, H. P., Patil, C. D., Salunkhe, R. B., Suryawanshi, R. K., Salunke, B. K., Patil, S. V. (2014). Phytolatex synthesized gold nanoparticles as novel agent to enhance sun protection factor of commercial sunscreens. Int. J. Cosmet. Sci., 36, 571-578.doi: 10.1111/ics.12158.

[20] Gonnelli, C., Giordano, C., Fontani, U., Salvatici, M.C., Ristori, S. (2018). Green Synthesis of Gold Nanoparticles from Extracts of Cucurbita pepo L. Leaves: Insights on the Role of Plant Ageing in Advances in Bionanomaterials. Lecture Notes in Bioengineering(Eds. S. Piotto, F. Rossi, S. Concilio, E. Reverchon, G. Cattaneo), Springer, Cham., pp. 155-174. doi: 10.1007/978-3-319-62027-5_14.

[21] Harti, A. S., Sulisetyawati, S. D., Murharyati, A., Oktariani, M. (2016). The Effectiveness of Snail Slime and Chitosan in Wound Healing. Int. J. Pharma Med. Biol. Sci., 5(1), 76-80. doi:10.18178/ijpmbs.5.1.76-80.

[22] Hatuikulipi, T. N., Kouachi, M., Bouchetob, L. E., Naimi, D. (2016). Preventive effect of Helix aspersa slime against experimentally chemo-induced colitis in rat. Der Pharmacia Lettre, 8(13),200-206.

[23] Conte, R. (2016). Recent advances on nano delivery of Helix mucus pharmacologically active components. Int. J. Nano Dimens., 7(3), 181-185. doi: 10.7508/ijnd.2016.03.001.

[24] Ellijimi, C., Hammouda, M. B., Othman, H., Moslah, W., Jebali, J., Mabrouk, H. B., Morjen, M., Haoues, M., Luis, J., Marrakchi, N., Essafi-Benkhadir, K., Srairi-Abid, N. (2018). Helix aspersa maxima 
mucus exhibits antimelanogenic and antitumoral effects against melanoma cells.Biomed Pharmacother, 101, 871-880. doi:10.1016/j.biopha.2018.03.020.

[25] Guillen Fabi, S., Cohen, J. L., Peterson, J. D., Kiripolsky, M. G., Goldman, M. P. (2013). The Effects of Filtrate of the Secretion of the Cryptomphalus aspersa on Photoaged Skin. J Drugs Dermatol, 12(4), 453-457-

[26] Tribo-Boixareu, M.J., Parrado-Romero, C., Rais, B., Reyes, E., Gonzalez, S. (2009). Clinical and histological efficacy of a secretion of the mollusk Cryptomphalus aspersa in the treatment of cutaneous photoaging. Cosmet. Dermatol., 22(5), 247-252.

[27] Greistorfer, S., Klepal, W., Cyran, N., Gugumuck, A., Rudoll, L., Suppan, J., von Byern, J. (2017). Snail mucus - glandular origin and composition in Helix pomatia. Zoology, 122, 126-138. doi:10.1016/j.zool.2017.05.001.

[28] Gubitosa, J., Rizzi, V., Fini, P., Cosma, P. (2019). Hair Care Cosmetics: From Traditional Shampoo to Solid Clay and Herbal Shampoo, A Review. Cosmetics, 6(1), 13-28. doi:10.3390/cosmetics6010013;

[29] Soto, M. L., Parada, M., Falqué, E., Domínguez, H. (2018). Personal-Care Products Formulated with Natural Antioxidant Extracts.Cosmetics, 5(1), 13-23. doi: 10.3390/cosmetics5010013.

[30] El Backly, R., Ulivi, V., Tonachini, L., Cancedda, R., Descalzi, F., Mastrogiacomo, M. (2011). Platelet Lysate Induces In Vitro Wound Healing of Human Keratinocytes Associated with a Strong Proinflammatory Response. Tissue Eng Pt, 17(13-14), 1787-1800.doi: 10.1089/ten.tea.2010.0729.

[31] Yin, X., Chen, S., Wu, A. (2010). Green chemistry synthesis of gold nanoparticles using lactic acid as a reducing agent. Micro Nano Lett, 5(5), 270-273. doi: 10.1049/mnl.2010.0117.

[32] Golmoraj, V. E., Khoshayand, M. R., Amini, M., Moghadamd, K. M., Amin, G., Shahverdi, A. R. (2011). The surface chemistry and stability of gold nanoparticles prepared using methanol extract of Eucalyptus camaldulensis. J. Exp. Nanosci., 6(2), 200-208.doi: 10.1080/17458080.2010.489581.

[33] Skingsley, D. R., White, A. J., Weston, A. (2000). Analysis of pulmonate mucus by infrared spectroscopy. J. Mollus. Stud., 66(3), 363-371. doi:10.1093/mollus/66.3.363.

[34] Tan, Y.W., Li, Y.F., Zhu, D.B. (2002). Fabrication of gold nanoparticles using a trithiol (thiocyanuric acid) as the capping agent.Langmuir, 18(8), 3392-3397. doi: 10.1021/la011612f.

[35] Li, X.H., Li, Y.C., Tan, Y.W., Yang, C.H., Li, Y.F. (2004). Self-assembly of gold nanoparticles prepared with 3,4-ethylenedioxythiophene as reductant. J. Phys. Chem. B, 108,5192-5199. doi: 10.1021/jp0356618.

[36] Xie, J.P., Lee, J.Y., Wang, D.I.C. (2007). Synthesis of single-crystalline gold nanoplates in aqueous solutions through biomineralization by serum albumin protein. J. Phys. Chem. C, 111, 10226-10232. doi: 10.1021/jp0719715.

[37] Casaletto, M. P., Longo, A., Martorana, A., Prestianni, A., Venezia, A.M. (2006). XPS study of supported gold catalysts: the role of $\mathrm{Au}-0$ and $\mathrm{Au}+$ delta species as active sites. Surf Interface Anal. 2006, 38(4), 215218. doi: $10.1002 /$ sia.2180.

[38] Love, C. S., Chechik, V., Smith, D. K., Wilson, K., Ashworth, I., Brennan, C. (2005). Synthesis of gold nanoparticles within a supramolecular gel-phase network. Chem. Commun., 15, 1971-1973.doi: 10.1039/b418190e.

[39] Sylvestre, J. P., Poulin, S., Kabashin, A. V., Sacher, E., Meunier, M., Luong, J. H. T. (2004). Surface chemistry of gold nanoparticles produced by laser ablation in aqueous media. J. Phys. Chem. B, 108(43), 16864-16869. doi: 10.1021/jp047134+.

[40] Fujigaya, T., Kim, C.R., Hamasaki, Y., Nakashima, N. (2016). Growth and Deposition of Au Nanoclusters on Polymer-wrapped Graphene and Their Oxygen Reduction Activity. Sci. Rep., 6, 21314. 
doi:10.1038/srep21314.

[41] Engin, S., Trouillet, V., Franz, C. M., Welle, A., Bruns, M., Wedlich, D. (2010). Benzylguanine Thiol Self-Assembled Mono layers for the Immobilization of SNAP-tag Proteins on Microcontact-Printed Surface Structures. Langmuir, 26(9), 6097-6101. doi:10.1021/la904829y.

[42] Artyushkova, K., Atanassov, P. (2013). X-Ray Photoelectron Spectroscopy for Characterization of Bionanocomposite Functional Materials for Energy-Harvesting Technologies. ChemPhysChem, 14(10), 2071-2080. doi: $10.1002 /$ cphc.201300037.

[43] Fanelli, F., Fracassi, F., Lapenna, A., Angarano, V., Palazzo, G., Mallardi, A. (2018). Atmospheric Pressure Cold Plasma: A Friendly Environment for Dry Enzymes. Adv. Mat. Interfaces, 5(24),1801373. doi: 10.1002/admi.201801373.

[44] Glassford, S. E., Byrne, B., Kazarian, S. G. (2013). Recent applications of ATR FTIR spectroscopy and imaging to proteins.Biochim Biophys Acta, 1834(12), 2849-2858. doi:10.1016/j.bbapap.2013.07.015.

[45] Barth, A. (2007). Infrared spectroscopy of proteins.Biochim. Biophys. Acta, 1767(9), 1073-1101. doi:10.1016/j.bbabio.2007.06.004.

[46] Akturk, O., Kismet, K., Yasti, A. C., Kuru, S., Duymus, M. E., Kaya, F., Caydere, M., Hucumenoglu, S., Keskin, D. (2016). Collagen/gold nanoparticle nanocomposites: A potential skin wound healing biomaterial.J. Biomat. Appl., 31(2), 283-301. doi:10.1177/0885328216644536.

[47] Pascale, R., Bianco, G., Cataldi, T. R. I., Schmitt Kopplin, P., Bosco, F., Vignola, L., Uhl, J., Lucio, M., Milella, L. (2018). Mass spectrometry-based phytochemical screening for hypoglycemic activity of Fagioli di Sarconi beans (Phaseolus vulgaris L.). Food Chem., 242, 497-504. doi: 10.1016/j.foodchem.2017.09.091.

[48] Dolashka, P., Dolashki, A., Velkova, L., Stevanovic, S., Molin, L., Traldi, P., Velikova, R., Voelter, W. (2015). Bioactive compounds isolated from garden snails. J. BioSci. Biotechnol., SE/ONLINE , 147-155.

[49] D'Alessio, S., Gerasi, L., Blasi, F. (2008). uPAR-deficient mouse keratinocytes fail to produce EGFR-dependent laminin-5, affecting migration in vivo and in vitro. J. Cell Sci., 121, 3922-3932.doi: $10.1242 /$ jcs.037549.

[50] Blasi, F., Carmeliet, P. (2002). uPAR: A versatile signalling orchestrator. Nat Rev Mol Cell Bio, 3(12), 932-943. doi:10.1038/nrm977.

[51] Wilson, H.M. (2010). Macrophages heterogeneity in atherosclerosis - implications for therapy. J. Cell. Molec. Med., 14(8), 2055-2065. doi: 10.1111/j.1582-4934.2010.01121.x.

[52] Talekar, M., Tran, T., Amiji, M. (2015). Translational Nano-Medicines: Targeted Therapeutic Delivery for Cancer and Inflammatory Diseases. AAPS Journal, 17(4), 813-827. doi:10.1208/s12248-015-9772-2.

[53] Mirza, R.E., Fang, M.M., Ennis, W.J., Koh, T.J. (2013). Blocking Interleukin-1 beta Induces a HealingAssociated Wound Macrophage Phenotype and Improves Healing in Type 2 Diabetes.Diabetes. 2013, 62(7), 2579-2587. doi: 10.2337/db12-1450.

[54] Sumbayev, V.V., Yasinska, I.M., Garcia, C.P., Gilliland, D., Lall, G.S., Gibbs, B.F., Bonsall, D.R., Varani, L., Rossi, F., Calzolai, L. (2013). Gold Nanoparticles Downregulate Interleukin-1-Induced Pro-Inflammatory Responses. Small, 9(3), 472-477. doi:10.1002/smll.201201528.

\section{Figure Captions}

Figure 1: Camera Pictures of a $\mathrm{HAuCl}_{4}$ solution at $4.0 \times 10^{-4} \mathrm{M}$ in absence (1) and presence (2) of SS (A); UV-Vis spectra of solutions containing AuNPs (diluted 1:5), observing the typical SPR, collected at different incubation time from the mixture of $\mathrm{HAuCl}_{4}$ and SS (B); Time evolution of SPR absorbance intensity $\left(\mathrm{A}_{\max }\right)$ versus the related FWHM (C); Time evolution of SPR wavelength registered in correspondence of the $A_{\max }$ versus the SPR FWHM (D). 
Figure 2: FESEM images of AuNPs-SS (A, B ); AuNPs size distribution determined from FESEM images (500 particles) (C).

Figure 3: High resolution XPS spectra of the AuNPs-SS and their relative curve-fitting: Au 4f (A), C 1s (B), O 1s (C), N 1s spectra (D).

Figure 4: ATR-FTIR spectra of SS, AuNPs-SS and SS after the AuNPs formation.

Figure 5: Positive ion MALDI-FT-ICR MS of the snail slime (A ) and AuNPs snail slime (B ) samples with DHB as matrix

Figure 6: Effect of pH on SPR wavelength position and FWHM (A ) and on ל-potential values (B ).

Figure 7: Confluent monolayers of NCTC2544 were wounded by manually scratching as described in Materials and Methods and incubated with increasing concentrations of AuNPs-SS. Representative pictures of the wound gap taken at $0 \mathrm{~h}, 24 \mathrm{~h}$ and $48 \mathrm{~h}$ (A) ; Histograms reported the percentage of gap closure compared with time $0 \mathrm{~h}(\mathbf{B})$; histogram representing viable cells (trypan blue negative) counted with the aid of a Burker chamber, as described in $\mathrm{M} \& \mathrm{M}(\mathbf{C})$; Western blot analysis of uPAR levels quantified by densitometric analysis; GAPDH was also examined to ensure equal loading of samples in each lane. For all the panels in the figure, data in the graphs represent the mean $\pm \mathrm{SD}$ of at least three independent triplicate experiments. Asterisks $\left({ }^{*} \mathrm{p}<0.05\right)$ indicate significant differences of AuNPs-SS treated cells, from CTRL (untreated)(D) .

Figure 8: Optical images of untreated, LPS treated, or AuNPs-SS treated cells (A); histogram representing viable cells(B); RT-PCR analysis of mRNA levels of IL1 $\beta$, IL6, iNOS in cells treated as in (A) (C) . Amplification of cDNA was carried out for 30 cycles and $\beta$-actin was used as the reference gene; results were from a typical experiment out of three. Densitometric analysis of the expression gene levels is reported at the bottom. Significance was assessed by one-way ANOVA test followed by Newman-Keuls post test. Error bars indicate mean $\pm \mathrm{SD}$; Asterisks $\left({ }^{*} \mathrm{p}<0.05\right)$ indicate significant differences of LPS+AuNPs-SS treated cells from LPS, while Number sign $(\# \mathrm{p}<0.05)$ indicates significant differences from CTRL (D) western blot analysis of iNOS and p65. Histograms on the right represent densitometric analysis of the iNOS and p65 protein levels. Asterisks $\left({ }^{*} \mathrm{p}<0.05\right)$ indicate significant differences of LPS+AuNPs-SS treated cells from LPS. Results were from a typical experiment out of three.

\section{Hosted file}

Figure 1.docx available at https://authorea.com/users/331555/articles/458202-biomoleculesfrom-snail-mucus-helix-aspersa-conjugate-gold-nanoparticles-exhibiting-potential-woundhealing-and-anti-inflammatory-activity

\section{Hosted file}

Figure 2.docx available at https://authorea.com/users/331555/articles/458202-biomoleculesfrom-snail-mucus-helix-aspersa-conjugate-gold-nanoparticles-exhibiting-potential-woundhealing-and-anti-inflammatory-activity

\section{Hosted file}

Figure 3.docx available at https://authorea.com/users/331555/articles/458202-biomoleculesfrom-snail-mucus-helix-aspersa-conjugate-gold-nanoparticles-exhibiting-potential-woundhealing-and-anti-inflammatory-activity

\section{Hosted file}

Figure 4.docx available at https://authorea.com/users/331555/articles/458202-biomoleculesfrom-snail-mucus-helix-aspersa-conjugate-gold-nanoparticles-exhibiting-potential-woundhealing-and-anti-inflammatory-activity

\section{Hosted file}


Figure 5.docx available at https://authorea.com/users/331555/articles/458202-biomoleculesfrom-snail-mucus-helix-aspersa-conjugate-gold-nanoparticles-exhibiting-potential-woundhealing-and-anti-inflammatory-activity

\section{Hosted file}

Figure 6.docx available at https://authorea.com/users/331555/articles/458202-biomoleculesfrom-snail-mucus-helix-aspersa-conjugate-gold-nanoparticles-exhibiting-potential-woundhealing-and-anti-inflammatory-activity

\section{Hosted file}

Figure 7.docx available at https://authorea.com/users/331555/articles/458202-biomoleculesfrom-snail-mucus-helix-aspersa-conjugate-gold-nanoparticles-exhibiting-potential-woundhealing-and-anti-inflammatory-activity

\section{Hosted file}

Figure 8.docx available at https://authorea.com/users/331555/articles/458202-biomoleculesfrom-snail-mucus-helix-aspersa-conjugate-gold-nanoparticles-exhibiting-potential-woundhealing-and-anti-inflammatory-activity 